\title{
Sensibilidad foveal en ojos con edema macular diabético con y sin engrosamiento temporal perifoveal
}

\author{
Foveal sensitivity in eyes with diabetic macular edema with and without temporal \\ perifoveal thickening \\ Virgilio Lima-Gómez ${ }^{*}$, Yatzul Z. García-Rubio ${ }^{1}$, Selma A. Somilleda-Ventura² y Dulce M. \\ Razo-Blanco-Hernández ${ }^{3}$ \\ ${ }^{1}$ Servicio de Oftalmología; ${ }^{2}$ Dirección de Investigación; ${ }^{3}$ División de Investigación. Hospital Juárez de México, Ciudad de México, México
}

\begin{abstract}
Resumen
Objetivo: Comparar la correlación entre la sensibilidad foveal y la agudeza visual en ojos con edema macular diabético, con y sin engrosamiento temporal perifoveal, característica asociada con mala respuesta terapéutica. Método: Estudio observacional, prospectivo, comparativo, transversal, en diabéticos con edema macular. Se comparó la correlación entre la sensibilidad foveal y la agudeza visual entre ojos sin (grupo 1) y con (grupo 2) engrosamiento temporal perifoveal (Rho de Spearman). Mediante regresión múltiple se determinó la contribución de la sensibilidad a los cambios de agudeza visual. Se evaluaron también los grosores del punto central y temporal perifoveal, y el volumen macular. Resultados: 60 ojos del grupo 1, 29 ojos del grupo 2. El promedio de sensibilidad no difirió entre grupos (30.0 \pm 0.59 vs. $28.4 \pm 1.05 \mathrm{~dB} ; p=0.2$ ), pero la correlación entre sensibilidad y agudeza visual sí (grupo 1, rho: -0.41; grupo 2, rho: -0.25 ). En el grupo 1, la sensibilidad fue la variable explicativa de la agudeza visual (beta: -0.52); en el grupo 2, ninguna variable se incluyó en el modelo y el grosor temporal perifoveal correlacionó inversamente con la sensibilidad (rho: -0.60). Conclusiones: el engrosamiento temporal perifoveal reduce la correlación entre la agudeza visual y la sensibilidad foveal en ojos con edema macular diabético.
\end{abstract}

PALABRAS CLAVE: Agudeza visual. Edema macular diabético. Engrosamiento temporal perifoveal. Retinopatía diabética. Sensibilidad foveal.
Abstract
Background: Visual improvement after photocoagulation in focal diabetic macular edema is more frequent when temporal perifoveal area is not affected; functional status before treatment could be worse in eyes with perifoveal temporal thickening. The correlation between visual acuity and foveal sensitivity (usually significant), would allow to compare macular function. Objective: To compare the correlation between retinal sensitivity and visual acuity in eyes with diabetic macular edema, with and without temporal perifoveal thickening. Method: Non-experimental, retrospective, comparative, cross-sectional study in type 2 diabetics with macular edema. The correlation between foveal sensitivity and visual acuity was compared in eyes without temporal perifoveal thickening (group 1) and eyes with it (group 2). Multiple regression analysis was used to identify the con- tribution of foveal sensitivity to the changes of visual acuity; other variables were: center point thickness, center field thickness, temporal perifoveal thickness and macular volume. Results: 60 eyes in group 1, 29 eyes in group 2. Mean sensitivity did no differ between groups (30.0 \pm 0.59 vs. $28.4 \pm 1.05 \mathrm{~dB} ; p=0.2)$, but the correlation between sensitivity and visual acuity did it (group 1, rho: -0.41 ; group 2, rho: -0.25 ). In group 1, foveal sensitivity was the only explaining variable of the regression model (beta: -0.52), in group 2 no one explaining variable was included and temporal perifoveal thickness had a negative

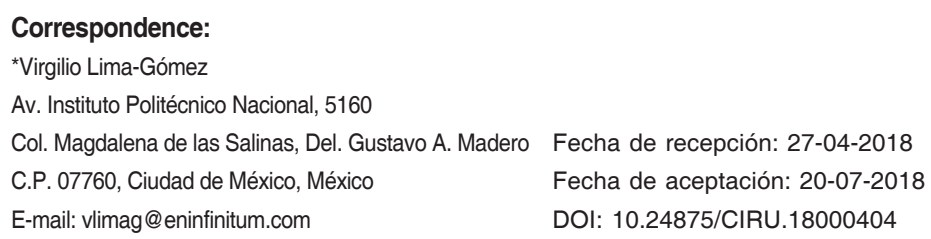
Cir Cir. 2019;87:34-39
Contents available at PubMed www.cirugiaycirujanos.com 
correlation with foveal sensitivity (rho: -0.60 ). Conclusions: Temporal perifoveal thickening reduces the correlation between foveal sensitivity and visual acuity in eyes with diabetic macular edema.

KEY WORDS: Diabetic macular edema. Diabetic retinopathy. Foveal sensitivity. Temporal perifoveal thickening. Visual acuity.

\section{Antecedentes}

La causa más común de pérdida visual en diabéticos con retinopatía es el edema macular', un engrosamiento de la zona con mejor función en la retina ${ }^{2}$. Esta enfermedad se diagnostica clínicamente, y su magnitud y extensión se cuantifican con herramientas de imagen como la tomografía de coherencia óptica $(\mathrm{TCO})^{3}$. El mapa rápido macular de la TCO divide la mácula en nueve campos, uno central y ocho en dos anillos circundantes $^{4}$, y permite identificar objetivamente el edema macular diabético con afectación central ${ }^{5}$.

Cuando se afecta el centro macular, el riesgo de pérdida visual aumenta, pero el grosor del punto central solo tiene una correlación de 0.59 con la agudeza visual $(\mathrm{AV})^{6}$. Una variable funcional que también correlaciona con la AV (rho: 0.49) es la sensibilidad retiniana (el nivel mínimo de iluminación detectable sobre un fondo iluminado), que disminuye cuando la AV es $<20 / 40^{7}$.

Una condición que limita la mejoría visual después de la fotocoagulación en ojos con edema focal es el engrosamiento temporal perifoveali; ${ }^{8}$ los ojos que lo presentan tienen un promedio de volumen macular más alto ${ }^{9}$ y requieren una mayor reducción de este para mejorar la visión.

Esta respuesta podría asociarse con un estado funcional distinto previo al tratamiento, que restringiera la mejoría visual aunque el grosor retiniano disminuyera, pero se desconoce la contribución que el grosor temporal perifoveal pudiera tener sobre la AV y la sensibilidad foveal. Dado que otras variables anatómicas tienen una correlación modesta con la AV, se desarrolló un estudio para determinar la contribución de la sensibilidad como variable explicativa de la AV en ojos con edema macular diabético focal, con y sin engrosamiento temporal perifoveal.

\section{Método}

Se realizó un estudio observacional, retrospectivo, comparativo y transversal. La población objetivo fueron sujetos con diabetes tipo 2 con edema macular focal de Ciudad de México y su área metropolitana. La muestra se obtuvo de pacientes atendidos en un hospital general del 1 de febrero de 2013 al 4 de

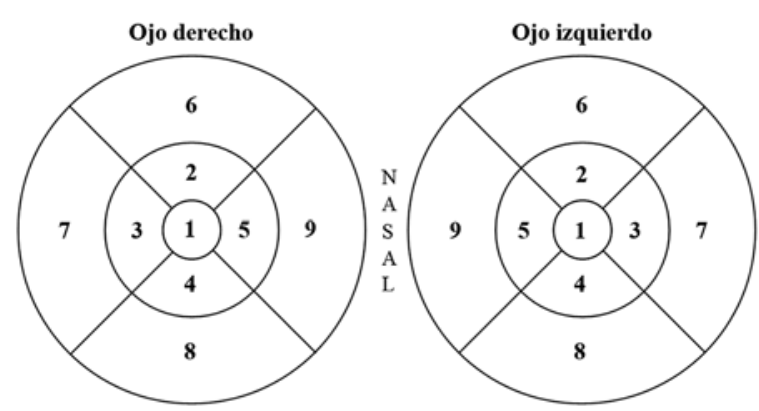

Figura 1. Campos del mapa rápido macular de tomografía de coherencia óptica.

marzo de 2014; se realizó un muestreo secuencial, no aleatorizado determinado por tiempo. El estudio fue autorizado por las comisiones de investigación y ética en investigación del hospital donde se realizó.

Se incluyeron sujetos con edad de 25 a 80 años, de ambos sexos, con cualquier grado de retinopatía no proliferativa, edema macular clínicamente significativo sin tratamiento previo, filtración angiográfica focal, fijación visual central y estable, que contaran con registro de la AV mejor corregida, campimetría macular y un mapa rápido macular de TCO. Se excluyeron los sujetos con cualquier otra enfermedad que redujera la AV corregida, patrón de engrosamiento por TCO de tipo cistoide, por tracción o con desprendimiento de retina neurosensorial, quienes tenían estudios de calidad deficiente y aquellos en quienes los datos de la fuente estuvieran incompletos.

De cada ojo evaluado se registraron las siguientes variables antes del tratamiento: $\mathrm{AV}$ corregida bajo refracción subjetiva, sensibilidad foveal medida con el campímetro Humphrey (Zeiss Meditec, Dublin, CA, EE.UU.) mediante la prueba de umbral macular, con un estímulo blanco de tamaño III sobre fondo blanco, y grosor retiniano medido bajo midriasis con la prueba de mapa rápido macular de $6 \mathrm{~mm}$ del equipo de TCO Stratus (Carl Zeiss Meditec, Inc., Dublin, CA, EE.UU., versión de software 4.01).

La AV se midió en logaritmo del ángulo mínimo de resolución (logMAR) y la sensibilidad foveal (calculada automáticamente por el equipo) se midió en decibeles $(\mathrm{dB})$. Los grosores del punto central y de los campos central (campo 1) y temporal perifoveal (campo 7) se midieron en micras ( $\mu \mathrm{m})$ (Fig. 1), y el volumen macular se midió en milímetros cúbicos $\left(\mathrm{mm}^{3}\right)$, de acuerdo con el cálculo automático del equipo ${ }^{10}$. 
Los ojos se asignaron a uno de dos grupos: $\sin$ engrosamiento temporal perifoveal (grupo 1) o con engrosamiento temporal perifoveal (grupo 2), que se definió como un grosor retiniano $>259 \mu \mathrm{m}$ en el campo temporal externo (7) del mapa rápido macular (Fig. 1), que supera por dos desviaciones estándar al promedio en sujetos sin retinopatía en la población de referencia ${ }^{11}$.

Todas las variables se compararon entre los grupos con la prueba $U$ de Mann-Whitney. Se realizó un análisis de regresión múltiple, que consideró como variable dependiente la AV y como variables explicativas los grosores del punto central, de los campos central y temporal perifoveal, el volumen macular y la sensibilidad foveal.

En cada grupo se identificó la correlación entre las variables mediante la prueba rho de Spearman, y su contribución en el modelo de regresión para explicar el valor de la AV. Se consideró significativa una $p<0.05$. La información se almacenó y analizó con la versión 21 del programa SPSS para Windows.

\section{Resultados}

Se evaluaron 89 ojos de 60 pacientes con una edad de 26 a 76 años (promedio \pm desviación estándar: $57.8 \pm 0.8) ; 43$ eran de sexo femenino (48.3\%). El tiempo de evolución de la diabetes fue de 3 meses a 30 años $(13.9 \pm 0.6)$. Treinta y siete ojos pertenecían a pacientes tratados con insulina (41.6\%) y el resto a pacientes tratados con hipoglucemiantes orales. Cuarenta y dos ojos eran de pacientes con hipertensión arterial (47.2\%).

La AV logMAR fue de -0.12 a $1.0(0.20 \pm 0.02)$. El grado de retinopatía fue no proliferativa leve en 16 ojos $(18.0 \%)$, moderada en $65(73.0 \%)$ y grave en 8 (9.0\%). El grosor del punto central fue de 122 a $298 \mu \mathrm{m}(172.0 \pm 3.4)$, el del campo central fue de 146 a $287 \mu \mathrm{m}(202.9 \pm 3.2)$ y el temporal perifoveal fue de 199 a $312 \mu \mathrm{m}(248.3 \pm 2.8)$. El volumen macular fue de 6.08 a $8.86 \mathrm{~mm}^{3}(7.4 \pm 0.06)$. La sensibilidad foveal fue de 12 a $37 \mathrm{~dB}(29.5 \pm 0.5)$.

Sesenta ojos no presentaron engrosamiento temporal perifoveal y se asignaron al grupo 1 ; los 29 restantes se asignaron al grupo 2. La comparación de las variables sistémicas entre los grupos se presenta en la tabla 1, y la de las variables oculares se muestra en la tabla 2. El promedio del volumen macular del grupo 2 superó estadísticamente al del grupo 1 (7.89 \pm 0.08 vs. $7.15 \pm 0.07 ; p<0.001)$. El grupo 2 tuvo promedios del
Tabla 1. Comparación de las variables basales entre los grupos.

\begin{tabular}{lccc}
\hline Variable & $\begin{array}{c}\text { Grupo 1 } \\
(\mathbf{n = 6 0})\end{array}$ & $\begin{array}{c}\text { Grupo 2 } \\
(\mathbf{n = 2 9 )}\end{array}$ & $\mathbf{p}$ \\
\hline Edad (años) & 59.1 & 55.0 & $0.02^{*}$ \\
Duración de la diabetes (años) & 14.5 & 12.7 & $0.2^{*}$ \\
Glucemia (mg/dl) & 156.9 & 201.2 & $0.3^{*}$ \\
Colesterol total (mg/dl) & 196.0 & 242.4 & $0.07^{*}$ \\
Triglicéridos (mg/dl) & 214.7 & 246.0 & $0.5^{*}$ \\
Sexo femenino (\%) & 45.0 & 55.2 & $0.3^{\dagger}$ \\
Tratamiento con insulina (\%) & 38.3 & 48.3 & $0.3^{\dagger}$ \\
Hipertensión arterial (\%) & 41.7 & 58.6 & $0.13^{\dagger}$ \\
\hline $\begin{array}{l}\text { *U de Mann-Whitney. } \\
\text { †ji al cuadrado. }\end{array}$ & & &
\end{tabular}

Tabla 2. Comparación de las variables oculares entre grupos.

\begin{tabular}{lccc}
\hline Variable & $\begin{array}{c}\text { Grupo 1 } \\
(\mathbf{n = 6 0 )}\end{array}$ & $\begin{array}{c}\text { Grupo 2 } \\
(\mathbf{n = 2 9 )}\end{array}$ & $\mathbf{p}^{*}$ \\
\hline Agudeza visual (logMAR) & $0.18 \pm 0.19$ & $0.23 \pm 0.28$ & 0.9 \\
Presión intraocular (mmHg) & $13.7 \pm 0.35$ & $13.3 \pm 0.66$ & 0.2 \\
Grosor del campo central $(\mu \mathrm{m})$ & $199.2 \pm 3.72$ & $210.6 \pm 5.98$ & 0.1 \\
Grosor temporal perifoveal $(\mu \mathrm{m})$ & $232.9 \pm 1.95$ & $280 \pm 3.19$ & $<0.001$ \\
Grosor del punto central $(\mu \mathrm{m})$ & $170.1 \pm 4.38$ & $175.7 \pm 5.6$ & 0.2 \\
Volumen macular (mm³) & $7.15 \pm 0.07$ & $7.89 \pm 0.08$ & $<0.001$ \\
Sensibilidad foveal $(\mathrm{dB})$ & $30.0 \pm 0.59$ & $28.4 \pm 1.05$ & 0.2 \\
\hline *U de Mann-Whitney. & & &
\end{tabular}

grosor del punto central y del grosor del campo central mayores, y de AV y sensibilidad macular menores que el grupo 1, aunque sin diferencia estadística.

\section{Grupo 1}

La matriz de las correlaciones bivariadas entre las variables se presenta en la tabla 3. La AV tuvo correlación negativa con la sensibilidad foveal (Fig. 2), y no tuvo correlación con las variables anatómicas; el volumen macular, la variable anatómica más robusta, tuvo correlaciones positivas con los grosores del campo central, del punto central y temporal perifoveal, y no tuvo correlación con las variables funcionales.

El grosor del punto central tuvo correlaciones positivas con los grosores del campo central y temporal perifoveal, y con el volumen macular. El modelo de regresión múltiple solo incluyó la sensibilidad foveal comovariable explicativa de la AV $(p<0.001$, beta $=-0.52)$. 
Grupo 1

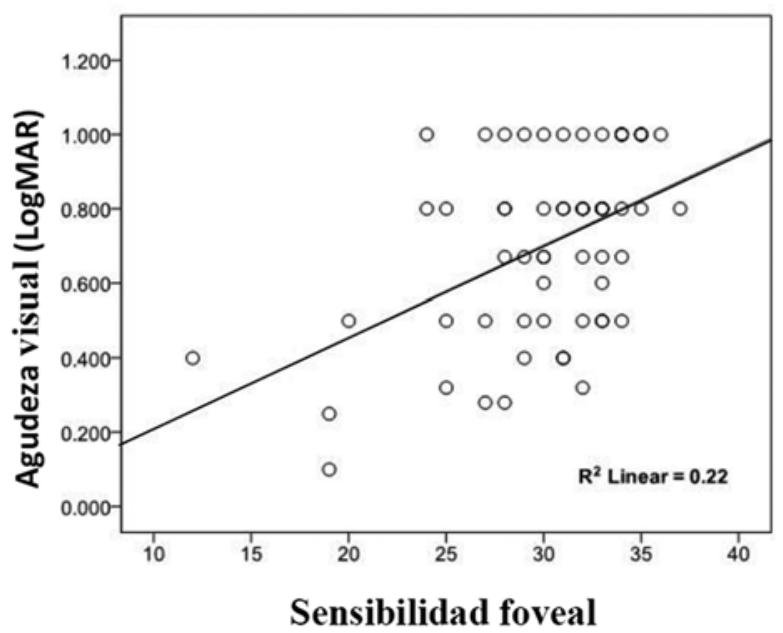

Grupo 2

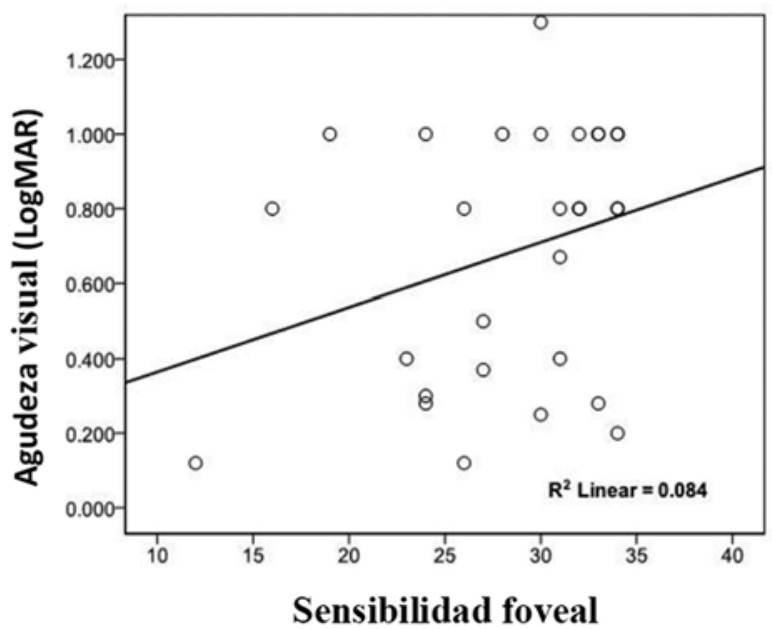

Figura 2. Correlación entre la sensibilidad foveal y la agudeza visual en ojos con edema macular diabético, con y sin engrosamiento temporal perifoveal.

Tabla 3. Matriz de correlaciones entre las variables oculares (rho de Spearman, por grupo).

\begin{tabular}{|c|c|c|c|c|c|c|c|c|c|c|c|c|}
\hline \multirow[t]{2}{*}{ Variable } & \multicolumn{6}{|c|}{ Grupo $1(n=60)$} & \multicolumn{6}{|c|}{ Grupo $2(n=29)$} \\
\hline & AV & GCC & GTP & GPC & VM & SF & AV & GCC & GTP & GPC & VM & SF \\
\hline AV & 1 & - & - & - & - & - & 1 & - & - & - & - & - \\
\hline GCC & 0.02 & 1 & - & - & - & - & 0.30 & 1 & - & - & - & - \\
\hline GTP & -0.06 & $0.42^{*}$ & 1 & - & - & - & 0.31 & 0.16 & 1 & - & - & - \\
\hline GPC & 0.08 & $0.97^{\star}$ & $0.35^{*}$ & 1 & - & - & 0.31 & 0.90 & 0.15 & 1 & - & - \\
\hline VM & 0.14 & $0.42^{*}$ & $0.83^{*}$ & $0.37^{\star}$ & 1 & - & 0.14 & 0.67 & 0.19 & $0.43^{*}$ & 1 & - \\
\hline SF & $-0.41^{*}$ & -0.03 & 0.13 & -0.13 & -0.04 & 1 & -0.25 & 0.09 & $-0.60^{*}$ & 0.01 & 0.11 & 1 \\
\hline
\end{tabular}

AV: agudeza visual; GCC: grosor del campo central; GPC: grosor del punto central; GTP: grosor temporal perifoveal; SF: sensibilidad foveal; VM: volumen macular.

Tabla 4. Características del modelo de regresión en el grupo 1.

\begin{tabular}{lc}
\hline Característica & $\mathbf{n}=\mathbf{6 0}$ \\
\hline Variable de desenlace & Agudeza visual \\
Variable predictora & Sensibilidad foveal \\
R & 0.52 \\
R cuadrada & 0.27 \\
R cuadrada corregida & 0.25 \\
Durbin-Watson & 0.63 \\
p (ANOVA) & $<0.001$ \\
Beta & -0.52 \\
\hline
\end{tabular}

Las variables anatómicas presentaron colinealidad, y su baja correlación con las funcionales las excluyó del modelo. Las características del modelo de regresión múltiple se presentan en la tabla 4.

\section{Grupo 2}

Las correlaciones bivariadas entre las variables se presentan en la tabla 3. La AV no tuvo correlaciones significativas. A diferencia del grupo 1, se encontró una correlación negativa significativa (rho: $-0.60 ; p=0.001$ ) entre una variable anatómica (grosor temporal perifoveal) y una funcional (sensibilidad foveal).

El volumen macular tuvo correlaciones positivas con los grosores del punto central y del campo central; estas últimas variables tuvieron la correlación más alta del grupo (rho: $0.9 ; p=0.001$ ). Aunque el promedio de la sensibilidad foveal en este grupo no fue estadísticamente menor que el del grupo 1, la correlación negativa de esta variable con el grosor temporal perifoveal (rho: $-0.60 ; p=0.001$ ) la excluyó del modelo de regresión; este modelo no incluyó alguna variable evaluada como explicativa de la AV. 


\section{Discusión}

El engrosamiento temporal perifoveal redujo el valor de la correlación entre la AV y la sensibilidad foveal en ojos con edema macular diabético.

Browning, et al. ${ }^{6}$ identificaron que la correlación más alta entre la AV y las variables medidas por TCO se presentó con el grosor del punto central (rho: 0.52). En nuestro estudio, la AV y el grosor del punto central tuvieron una correlación de 0.31 solo en el grupo 2, pero no fue significativa. El estudio de Browning, et al. ${ }^{6}$ no evaluó la sensibilidad foveal, que en el grupo 1 de esta muestra tuvo una correlación significativa con la AV y superó la de cualquier variable anatómica.

La sensibilidad retiniana puede disminuir en los sujetos con diabetes ${ }^{12}$. De Benedetto, et al. ${ }^{13}$ encontraron una reducción de la sensibilidad retiniana en sujetos diabéticos sin edema macular con un ligero aumento del grosor del campo central, mientras que Kothari, et al. ${ }^{14}$ reportaron que la sensibilidad retiniana se conservaba en ojos con edema macular diabético y patrón espongiforme en la TCO. Los resultados de ambos estudios divergen, pero ninguno evaluó el grosor temporal perifoveal, que no se había considerado una covariable; sin embargo, Michalska, et al. ${ }^{15}$ reportaron que, en ojos con edema macular diabético, la sensibilidad retiniana y el grosor temporal perifoveal no cambiaron 6 meses después de la fotocoagulación.

Los ojos del grupo 2 requirieron un estímulo lumínico un $45 \%$ mayor (10 vs. 14.45 apostilbs), aunque su promedio de sensibilidad foveal no difirió del grupo 1 (28.4 vs. $30.0 \mathrm{~dB}$ ); un tercio del valor de la sensibilidad foveal en el grupo 2 se explicó por el grosor temporal perifoveal $\left(R^{2}=0.33\right)$. La perifóvea temporal tiene características que podrían modificar la función foveal, como la ubicación del plexo capilar superficial en el límite externo de la capa de células ganglionares ${ }^{16,17}$, una menor latencia de la actividad eléctrica que la perifóvea nasal (tiempo implícito en el electrorretinograma: $30.2 \pm 3$ vs. $31 \pm 3 \mathrm{~ms} ; p=0.005)^{18} y$ que cuando exista fuga capilar en el área temporal se altere su flujo y cause una asimetría respecto al sector nasal' ${ }^{19}$.

La correlación negativa entre el grosor temporal perifoveal y la sensibilidad foveal podría indicar que la afectación de la mácula temporal reduce la función foveal, independientemente del grosor del punto central y del volumen macular. La correlación entre la AV y la sensibilidad foveal en el grupo 2 (rho: -0.25) no disminuyó por efecto de las variables anatómicas evaluadas comúnmente, ni se hubiera detectado por una comparación simple entre grupos, ya que los promedios de grosor del punto central y del campo central no difirieron entre ellos.

Entre las fortalezas del estudio se encuentran el centrado adecuado de los mapas para medir el grosor del punto central, que todos los pacientes tuvieron fijación central y estable, y la homogeneidad de la distribución del volumen macular. Una limitante fue que no se calculó un tamaño de muestra, por no existir información previa para estimar la diferencia entre grupos. Otra potencial limitante es que no se evaluó la unión entre los segmentos internos y externos de los fotorreceptores, ni la membrana limitante interna, que requerirían generaciones más recientes de TCO; aunque las alteraciones de estas capas se han descrito en edema difuso, sería conveniente identificar si son más comunes cuando el edema macular focal se localiza en el sector temporal perifoveal.

En resumen, el engrosamiento temporal perifoveal redujo la sensibilidad foveal lo suficiente para que los cambios de esta variable dejaran de explicar los de la AV en diabéticos con edema macular. En estudios futuros deberá evaluarse la consistencia de estos hallazgos y su efecto sobre el desenlace funcional después del tratamiento.

\section{Conclusión}

La correlación entre la AV y la sensibilidad foveal en ojos con edema macular diabético fue significativa únicamente en ojos sin engrosamiento temporal perifoveal.

\section{Conflicto de intereses}

Los autores declaran que no tienen conflicto de intereses alguno para el desarrollo y la publicación del trabajo.

\section{Responsabilidades éticas}

Protección de personas y animales. Los autores declaran que para esta investigación no se han realizado experimentos en seres humanos ni en animales.

Confidencialidad de los datos. Los autores declaran que han seguido los protocolos de su centro de trabajo sobre la publicación de datos de pacientes.

Derecho a la privacidad y consentimiento informado. Los autores han obtenido el consentimiento informado de los pacientes y/o sujetos referidos en el 


\section{artículo. Este documento obra en poder del autor de correspondencia.}

\section{Bibliografía}

1. Ding J, Wong TY. Current epidemiology of diabetic retinopathy and diabetic macular edema. Curr Diab Rep. 2012;12:346-54.

2. Danis RP, Scott IU, Qin H, Altaweel MM, Bressler NM, Bressler SB, et al Association of fluorescein angiographic features with visual acuity and with optical coherence tomographic and stereoscopic color fundus photographic features of diabetic macular edema in a randomized clinical trial. Retina. 2010;30:1627-37.

3. Cheung N, Mitchell P, Wong TY. Diabetic retinopathy. Lancet. 2010; 10:124-36.

4. Buabbud JC, Al-latayfeh MM, Sun JK. Optical coherence tomography imaging for diabetic retinopathy and macular edema. Curr Diab Rep. 2010;10:264-9.

5. Browning DJ. Interobserver variability in optical coherence tomography for macular edema. Am J Ophthalmol. 2004;137:1116-7.

6. Browning DJ, Glassman AR, Aiello LP, Beck RW, Brown DM, Fong DS et al. Relationship between optical coherence tomography-measured central retinal thickness and visual acuity in diabetic macular edema. Ophthalmology. 2007:114:525-36.

7. Lima-Gómez V, García-Rubio YZ, Razo Blanco-Hernández DM. Sensibilidad retiniana en el edema macular diabético, con y sin disminución de la agudeza visual. Gac Med Méx. 2014;150(Suppl 1):114-9.

8. Lima-Gómez V, Razo Blanco-Hernández DM. Características asociadas con la mejoría visual después de la fotocoagulación en edema macular diabético. Cir Cir. 2012;80:311-9.

9. Razo Blanco-Hernández DM, Vargas-Romero DM, Bermúdez-Zapata DA Lima- Gómez V. Modificación del volumen macular por engrosamiento del área temporal, en edema macular diabético focal. Rev Hosp Jua Mex. 2011;78:146-51.

10. Polito A, Del Borrello M, Isola M, Zemella N, Bandello F. Repeatability and reproducibility of fast macular thickness mapping with Stratus optical coherence tomography. Arch Ophthalmol. 2005;123:1330-7.

11. Lima-Gómez V, Osornio-Castro NA. Comparación del grosor retiniano en diabéticos sin retinopatía, con y sin fondo coroideo. Rev Mex Oftalmol. 2006:80:301-5

12. Cabezos I, Luque MJ, de Fez D, Moncho V, Camps V. Chromatic-achromatic perimetry in four clinic cases: glaucoma and diabetes. Indian $\mathrm{J}$ Ophthalmol. 2015;63:146-51.

13. De Benedetto U, Querques G, Lattanzio R, Borrelli E, Triolo G, Maestranzi $\mathrm{G}$, et al. Macular dysfunction is common in both type 1 and type 2 diabetic patients without macular edema. Retina. 2014; 34:2171-7.

14. Kothari AR, Raman RP, Sharma T, Gupta M, Laxmi G. Is there a correlation between structural alterations and retinal sensitivity in morphological patterns of diabetic macular edema? Indian J Ophthalmol. 2013; 61:230-2.

15. Michalska A, Dorecka M, Jackiewicz K, Miniewicz-Kurkowska J, Sobieraj R, Michalski M, et al. Evaluation of mean retinal sensitivity using MP-1 microperimeter in patients with diabetic macular edema before and after laser photocoagulation treatment. Pol Arch Med Wewn. 2013;123:98-104.

16. Iwasaki M, Inomata $\mathrm{H}$. Relation between superficial capillaries and foveal structures in the human retina. Invest Ophthalmol Vis Sci. 1986;27:1698-705.

17. Agarwal A. Gass' Atlas of macular diseases. $5^{\text {th }}$ ed. Edinburgh: Elsevier Saunders; 2012. p. 8.

18. Holm K, Lövestam Adrian M. In diabetic eyes, multifocal ERG reflects differences in function between the nasal part and the temporal part of the macula. Graefes Arch Clin Exp Ophthalmol. 2012;250:1143-8.

19. Hudson C, Flanagan JG, Turner GS, Chen HC, Rawji MH, McLeod D. Exaggerated relative nasal-temporal asymmetry of macular capillary blood flow in patients with clinically significant diabetic macular oedema. Br J Ophthalmol. 2005;89:142-6. 